\title{
Information-applied Technology in Crop Growth Models Based on LED Light Source Environment
}

\author{
Meng $\mathrm{Li}^{1, \mathrm{a}}$, Cong Zhang ${ }^{1}$, Liguo Tian $^{1}$ \\ ${ }^{1}$ Tianjin Key laboratory of Information Sensing \&Intelligent Control, Tianjin University of Technology \\ and Education, Tianjin 300222 China \\ alimeng-3260711@163.com
}

Keyword: LED Light Source; Crop Growth Models; Environmental Factors; Crop Growth Simulation System

\begin{abstract}
Currently, using crop growth model to study crop yield, quality, and its relationship with the relevant factors at home and abroad has become a hot research. This paper built a simulation system for crop growth based on LED light source environment, and select typical greenhouse crops to research the relationship between crop growth and light, temperature, humidity, carbon dioxide and other environmental factors. The crop growth model was set up using the relationship and computer simulation software was built based on crop model, so the growing status of the crop based on LED light source environment are shown both with dynamic image and database. The system makes the best of multimedia techniques and has favorable expansibility and human computer interaction function.
\end{abstract}

\section{Introduction}

Crop growth model which is called for crop models is a new field of study after the introduction of systems analysis methods and applications computer in Crop Science. Crop growth model is in study with the crop, based on farming systems and crop scientific principles to generalize and analysis the process of photosynthetic production, physiological processes, organs formation, yield and the relationship between the environment and technology. Then, a mathematical model is built to analysis quantitatively and simulates crop growth process on the computer. Crop model is a powerful tool for precise management in crop production, and has important theoretical significance and application value in facility crop production optimize management [1-2].

As we all know, light is an important environmental factor for crop growth and development, and the light source for plant development is mainly from solar radiation on Earth. In recent years, with the maturity and development of LED technology, LED light source has been successfully used in the field of artificial light, plant tissue culture, genetics breeding, plant factory and space agriculture, is considered to be the most promising artificial light in the field of Agriculture and Biology in 21st century, has a good development prospects [3].

Based on the influence of LED light for the state of crop growth, the simulation system for crop growth in LED light source environment is established. According to the various different stages characteristics of crop growth, the simulation system for crop growth is to control the environment parameters, such as temperature, light, moisture and other parameters. Then, a nonlinear system of equations with crop physiological development time scale is established to describe the growth of crops, thus design and development the crop growth simulation model with LED light source, and development a software of user-friendly interface, the model parameters and convenient data input and output convenient and good application.

\section{The research program of system}

Based on the system analysis of research results at home and abroad, using scientific testing methods, selecting the representative crop varieties, using LED as the light source, to establish the theoretical system and technical methods of crop growth model in LED light source environment. 
This paper is focus on exploration and research the relationship between crop growth and temperature, light, moisture, wind speed and other environmental factors. From the growth mechanism of the crop, a dynamic simulation model of crop growth in LED light source is established, and a computer simulation system is developed which using animation and digital display to simulate crop growth. The research program of system is shown in Figure.1.

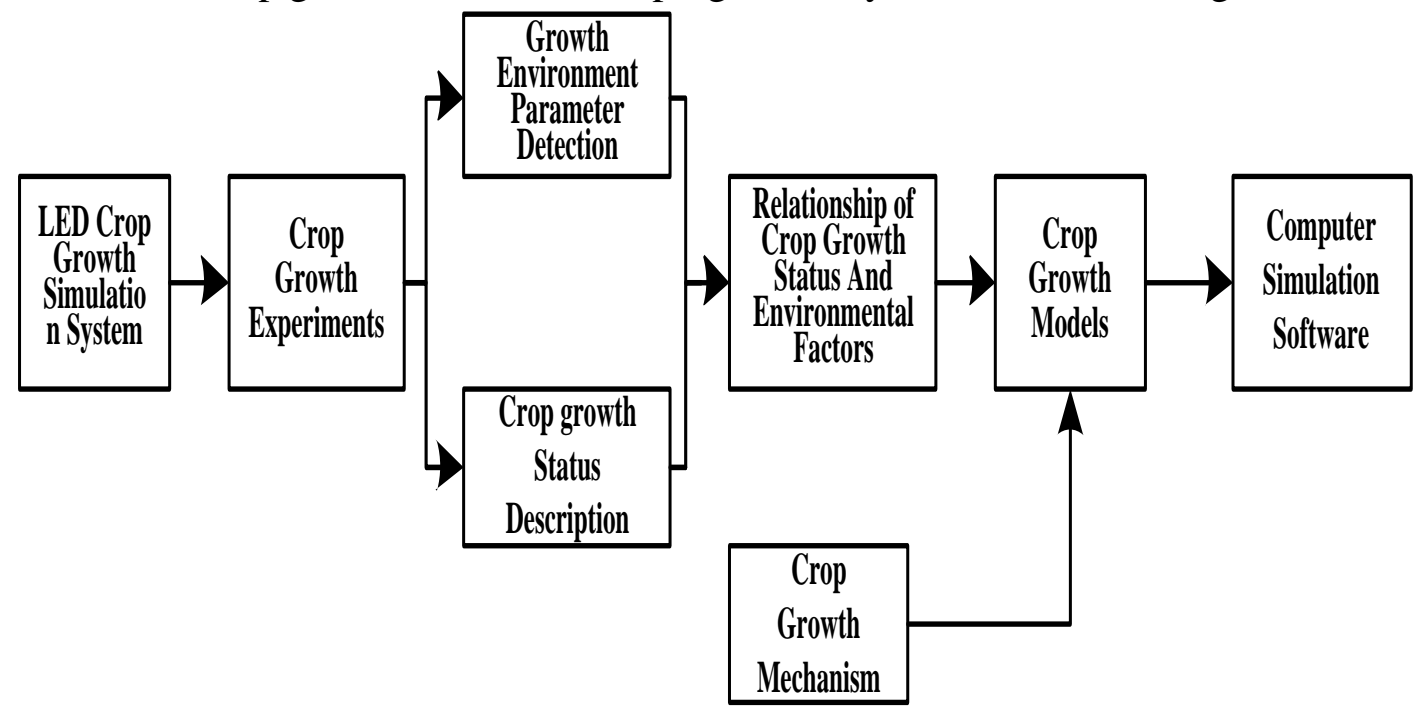

Fig. 1 The research program of system

\section{The design of simulation system for crop growth based on LED light}

The main function of simulation system for crop growth in LED light environment is provide optimum temperature, humidity, light intensity, and carbon dioxide levels and other climatic conditions for crops growth. The system is mainly contains of crop cultivation and control systems: the crop cultivation system for cultivation of crops is including box and auxiliary equipment, and the structural insulated box with the cabinet. The control system consists of upper and lower parts, the upper part realizes the man-machine interface to control and receive real-time environmental parameters from lower machine to upload the best environmental conditions parameters and crop need to send to the next crew, the next crew in accordance with established given parameter automatic control system to run crop cultivation; the main function of lower machine is to achieve the environmental parameters of the acquisition, while the serial communication with the host computer, according to the host command environment growth cabinet parameters are adjusted to ensure that the growth cabinet the crop is under optimal growth conditions, so as to achieve high-quality, high purpose, the overall design of the control system block diagram is shown in Figure.2.

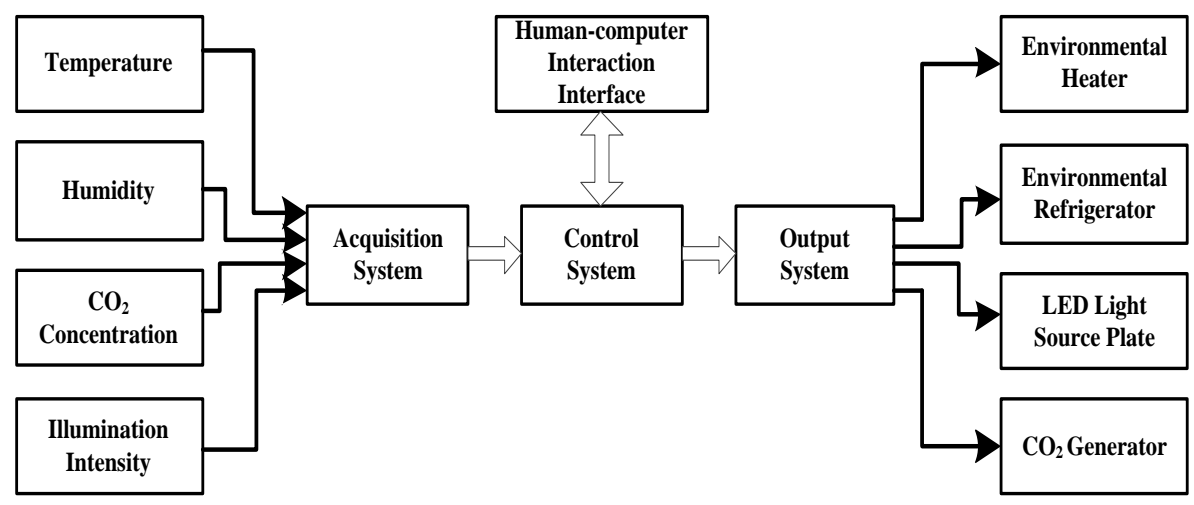

Fig. 2 The block diagram of control system 


\section{The relational model of crop growth and environmental factors}

Crop growth is a complex and constantly changing process, and contact with the outside environment interaction every moment in crop growth throughout the life cycle. The block diagram of the relationship of crop growth is shown in Figure.3. The photosynthesis, respiration and other characterization of the strength of crop growth metabolism are no necessary connection with environmental temperature and humidity, light, carbon dioxide and other environmental factors. The characterization of growing crop leaf area index, chlorophyll content, dry matter accumulation and temperature indicators humidity, wind speed, nutrients and other factors exist necessarily linked. The impact of changes in exploration and study of environmental factors on the growth of the state's crop itself, establish a relationship between the two models, thus providing data support for the establishment of the LED light source crop growth models.

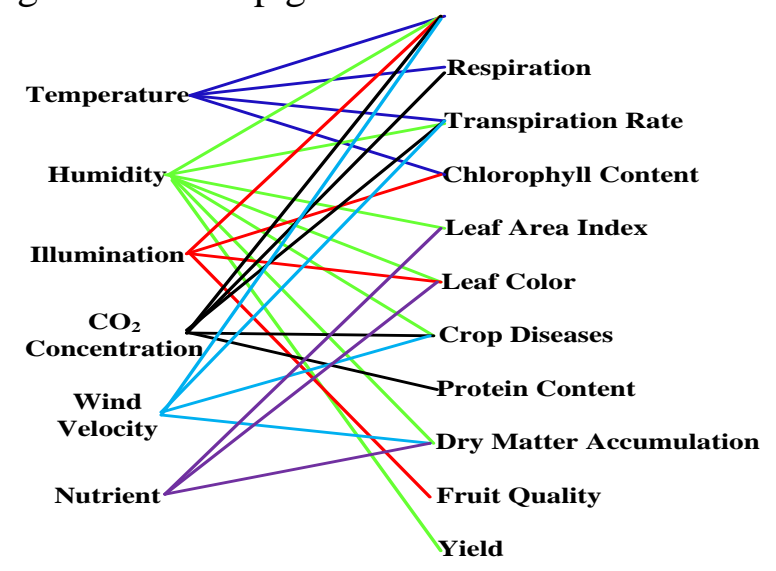

Fig. 3 The block diagram of the relationship of crop growth

\section{The achievement of crop growth models based on LED light source environmental}

According to the relationship between the different stages of crop growth and various environmental factors, a dynamic model is established to describe the growth and development processes of crop in LED light source environment. Using of computer technology, the crop growth modeling software is developed to simulate crop growth in LED light environments.

In the model, some different modules are include, such as data input and output modules, the environment module, crop morphological development module. The logic and program flow is shown in Figure.4. 


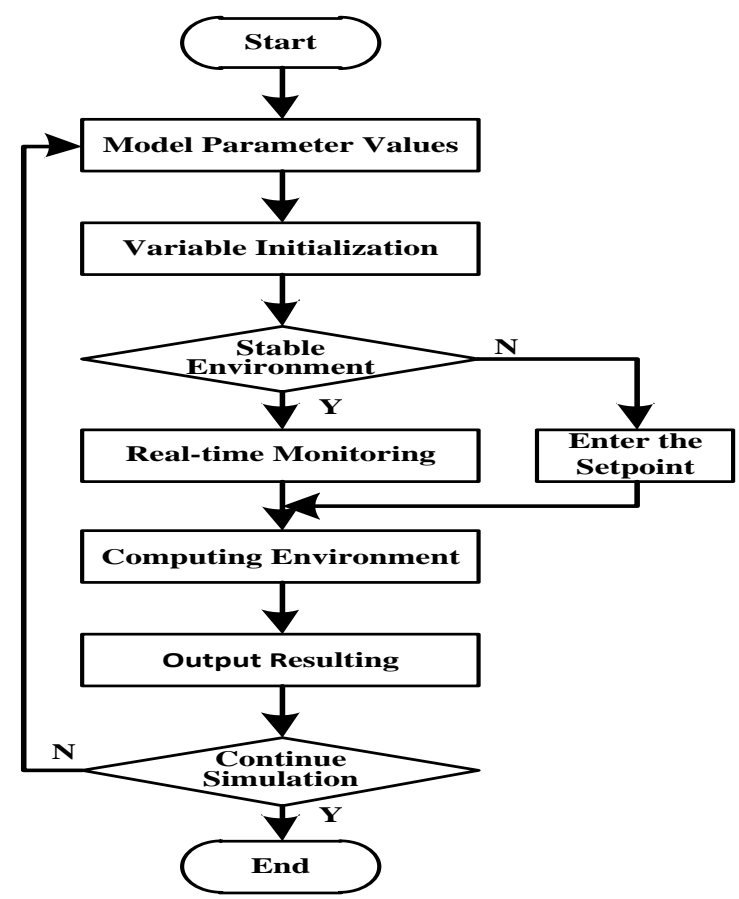

Fig.4 The logic and program flow

\section{Conclusions}

Currently, although crop growth model has been made considerable progress, but in some physiological and ecological mechanism is still subject to further study and further improved. In this paper, the simulation system for crop growth in LED light source environment is built to research of crop growth model. According to the relationship between crop growth and environmental factors, a crop growth model LED light in the environment is established, and computer simulation software is developed for research of crop model in further.

\section{Acknowledgement}

In this paper, the research was sponsored by the School-level Scientific Research Project of Tianjin University of Technology and Education (Project No.KJ13-02) and National High Technology Research and Development Program of China (863 Program, No.SS2013AA03120).

\section{References}

[1] Bo Ma, Jun-cang Tian. A Review on Crop Growth Simulation Model Research [J]. Water-saving irrigation, 20102 1-4.

[2] Ya-li Wang, Li-yuan He. A Review on the Research and Application of Crop Simulation Model [J]. Journal of Huazhong Agricultural University, 2005 24(5) 529-535.

[3] Jin Cui, Zhi-gang Xu,Xiu-ru Di. Applications and prospects of light emitting diode in plant protected culture [J]. Transactions of the CSAE, 2008 24(8) 249-253.

[4] Yang Hu, Sha Jiang,Jie Li. Effects of The Light Intensity and Quality on Plant Growth and Development[J]. Journal of Inner Mongolia Agricultural University, 2009 30(4) 296-303.

[5] Yong-liu Li, Wei-hong Luo. Review on research progress of greenhouse vegetable growth and development models [J]. Transactions of the CSAE, 2008 24(1) 307-311.

[6]Juan Yao, Tie-mei Liu. Study on Computer Simulation Softer ware Based on Crop Model [J]. Modern Agricultural Sciences and Technology, 201020 31-34. 